\title{
Treacher- Collins Syndrome unusually in siblings
}

\author{
Dr. Apeksha S. Dhole (Balpande) ${ }^{1}$, Dr. Bhavana Agrawal $^{2}$, Dr. Amit Parate ${ }^{3}$ \\ ${ }^{I}$ (Department Of Oral Medicine \& Radiology, VSPM Dental College, Nagpur/ MUHS, Nashik, India) \\ ${ }^{2}$ (Department Of Oral Medicine \& Radiology, Jodhapur Dental College General Hospital / Jodhapur, India) \\ ${ }^{3}$ (Department Of Oral Medicine \& Radiology, Government Dental College \& Hospital, Nagpur / MUHS, India)
}

\begin{abstract}
Treacher-Collins Syndrome (TCS) is a rare autosomal dominant disorder of craniofacial morphogenesis. The frequency of TCS is 1 in 50,000 live births. Approximately half of cases arise as a result of sporadic mutation; the rest are familial. TCS is caused by a mutation in a TCOFI gene on chromosomes 5q31.3-32. The severely affected persons show classic physical characteristics and mildly affected may escape the diagnosis. Till now cases with familial or genetic background with variable expression were reported. We have disscussed this syndrome unusually in siblings.
\end{abstract}

Keywords: Autosomal dominence, siblings, Treacher- Collins Syndrome (TCS),TCOFI gene.

\section{INTRODUCTION}

TCS is a rare autosomal dominant disorder characterised primarily by defects of structures derived from first and second branchial arches[1]. Online Mendelian Inheritance in Man (OMIM) number of TCS is 154500. Treacher Collins, a British ophthalmologist, in the year 1900 described essential features of this syndrome. It occurs in around 1 of every around 50,000 live births[2]. The condition has variable expressivity. The severity of clinical features often tends to be greater in subsequent generations of the same family. Approximately $60 \%$ of cases represents new mutations, and these often are associated with increased paternal age. Genetically, the treacle gene (TCOF 1) is mutated. It is found on chromosome 5q31.3-32 and encodes a serine/ alanine rich nucleolar phosphoprotein responsible for the craniofacial development [2].

\section{Case 1}

A 14 years old girl reported with chief complaint of crowding and proclined upper anteriors. When the patient was examined, she revealed characteristic bilateral facial abnormalites with downward slanting of eyes, depressed zygomatic arches, sunken cheek bones, coloboma of lower eyelid with deficient eyelashes medial to it, tongue -shaped extentions of hairs on cheek, retruded chin giving bird-like appearance. She has convex profile.External ear was normal with impaired hearing on left side and she didn't show any signs of mental retardation "Fig 1, 2, 3".

Intraoral examination revealed high arched palate, Angles class I type 1 and type 2 malocclusion with incompetent lips "Fig 4a, b".

Lateral cephalometric views revealed prominent antigonial notch, steep mandibular plane angle, hypoplastic mandible and acute nasolabial angle "Fig 5". PNS shows underdeveloped maxillay sinus, malar bones and hyperteloric orbits "Fig 6".

Based on clinical and radiographical findings she was diagnosed as TCS. The treatment was tailored to rehabilitate the oral cavity of the patient so as to aid her in proper mastication and aesthetics. She was accompanied with her younger brother who showed mild features of the syndrome.

\section{Case2-}

He was 12 years old boy with normal intelligence. On extraoral examination he was having slanting eyes, depressed zygomatic arches, sunken cheeks, coloboma of lower eyelid with very few eyelashes and tongue shaped extension of hair on cheek. External ear was abnormal with impaired hearing. Intraorally mild crowing in lower anterior region was noted "Fig 7, 8, 9". He was also dignosed as TCS on clinical examination. Patient was not willing for radiographic examination and any treatment.

Neither of their parents nor grandparents was affected with this syndrome. At the time of marriage their mother was 21 years and father was of 25 years old. Mother's age was 22 year old at first pregnacy and 24 years during second. These two cases show presence of TCS unusually in siblings without any familial background.

In our patients, the best part was their vision, mental functioning, speech were normal. Thus the prognosis was good. The patients were also planned to refer to medical geneticist at proper age for councelling as they have $50 \%$ chances of having affected baby. 


\section{DISCUSSION}

Treacher Collins syndrome was named after the British ophthalmologist Edward Treacher Collins (1862-1932), who described the essential features of this syndrome in 1900. However, some features of this syndrome were probably first described by Thomson and Toynbee in 1846-1847 and later by Berry (1889), who is usually given credit for its discovery. It is also called as Franceschetti-Zwahlen-Klein syndrome, based on extensive studies of mandibulofacial dysostosis published by the Swiss ophthalmologist Franceschetti and the geneticist Klein (1949).[3]

Lungarotti et al. (1987) described changes strikingly similar to those of vitamin A toxicity in both animals and humans in an infant born of a mother who took 2000 IU of vitamin A daily as a supplement during pregnancy[4]. Marsh et al. (1998) ,Li et al. (2009) and Bowman et al. (2012) suggested that the disorder results from mutations in the TCOF1 gene[5,6,7].

TCOF1 is the only gene currently known to be associated with TCS, a mutation in this gene being found in $90-95 \%$ of the individuals with TCS. TCOF1 codes for a relatively simple, nucleolar protein called Treacle. Mutations in TCOF1 lead to haploinsufficiency of the Treacle protein. Haploinsufficiency occurs when a diploid organism has only one functional copy of a gene, because the other copy is inactivated by a mutation. Thereby the other functional gene does not produce enough of a gene-product to have the expected function, resulting in an abnormal disease state. Haploinsufficiency of the Treacle protein leads to a depletion of the neural crest cell precursor, which leads to a reduced number of crest cells migrating to the first and second pharyngeal arches. These crest cells play an important role in the development of the craniofacial appearance. So down regulation of Treacle expression results in craniofacial defects and growth retardation [4].

TCS is inherited in an autosomal dominant manner and the penetrance of the affected gene is almost complete. On the other hand some recent investigations described some rare cases in which the penetrance in TCS was not complete. Causes may be a variable expressivity, an incomplete penetrance or germline mosaicism. Only $40 \%$ of the mutations are inherited. The remaining $60 \%$ is a result of a de novo mutation. In the outcome of the disease there is a lot of inter- and intrafamilial variability. This suggests that when an affected child is born, it is important to investigate the parents if the affected gene is present. It could be possible that a parent has a mild and undiagnosed TCS. In this case the risk of having another affected child is $50 \%$. If the parents do not have the affected gene, the recurrence risk appears to be low.

Although $40 \%$ of individuals diagnosed with autosomal dominant TCS have an affected parent, the family history may appear to be negative because of failure to recognize the mild expression of the disorder in family members or the rare occurrence of incomplete penetrance in a parent.

Diagnosis of TCS easily determined when a full expressivity of the syndrome occurs. Diagnostic problems may be encountered when only a minimal expression of facial features is present. The characteristic facies of the patients are often described as birdlike or fish-like in nature. Typical morphologic changes occur in TCS . Antimongoloid palpebral fissures with a coloboma of the outer portion of the lower lids, and deficiency of the eyelashes which was present in our cases. Hypoplasia of the facial bones, especially of the malar bones and mandible, the chin recedes with a large, down-turned mouth. Malformation of external ear, and occasionally of the middle and internal ears, The pinnae are often malformed, crumpled forward, or misplaced toward the angle of the mandible, Frequently, meatal atresia, external auditory canal stenosis or atresia, hypoplasia or agenesis of the malleus and the incus, monopodal stapes, ankylosis of stapes in the oval window, and absence of the middle ear and tympanic spaces are present, resulting in a conductive hearing loss. Extra ear tags and blind fistulas may develop anywhere between the tragus and the angle of the mouth. [4,5,8]

Macrostomia, high palate ( sometimes cleft) and abnormal position and malocclusion of the teeth. Atypical hair growth in the form of a tongue- shaped process of the hairline extending towards the cheeks. This is very rare feature which was present in our cases. Facial clefts may present in few cases. The nose appears large because of the lack of malar development and hypoplastic supraorbital ridges. The parotid glands are missing or hypoplastic.Pharyngeal hypoplasia is a constant finding. Intelligence is usually normal, developmental delay may be secondary to undiagnosed hearing loss.Patient may show few dysfunctional symptoms like hypoplasia and a retropositioned tongue,difficulties with swallowing and feeding (caused by musculoskeletal underdevelopment and a cleft palate), conductive hearing loss (caused by maldevelopment of the auditory canal and middle ear ossicles), impaired vision (caused by underdeveloped lateral orbit and extraocular muscles). Radiographic features are helpful in many cases in diagnosing this syndrome. The malar bones, zygomatic process of frontal bone, lateral pterygoid plates, paranasal sinuses, and mandibular condyles are hypoplastic. The mastoids are not pneumatized. The lateral margins of the orbits may be defective, and the orbits are hyperteloric. The cranial base is progressively kyphotic. The calvaria are essentially normal. The auditory ossicles are often absent, and the cochlea and vestibular apparatus may be deficient. The mandibular angle is more obtuse than normal and the ramus is deficient. The coronoid and condyloid processes are flat or aplastic[9]. 


\section{Laboratory Studies-}

Prenatal diagnosis of the syndrome can be done if family history is positive, by a) chorionic villus sampling between 10-12 weeks of pregnancy b) amniocentesis between 14- 18 weeks and c) high resolution ultrasound between 18-20 weeks of pregnancy.However it become difficult to diagnose where the fetus is mildly affected. While postnatal dignosis is by evaluation of clinical features and confirmation by DNA examination of affected gene[10].

\section{Treatment plan -}

Treatment of TCS is lengthy and requires a multidisciplinary approach focused on treatment of symptoms. Excellent outcomes are achievable through a comprehensive, well-coordinated and integrated treatment plans incorporating craniofacial surgeons, orthodontists, ophthalmologists, otolaryngologists and speech pathologists[10]. However, the results are often variable and may not be fully corrective, hence considerable effort should also be invested into examining therapeutic avenues of prevention. Dentist plays major role in managing patients psychology and speech by correcting oral symptoms.

\section{Conclusion-}

The major challenge in TCS patients are in terms of improving the prognosis of affected or at risk individuals reside in three key areas; early detection, repair and prevention. Dentist have major role in rehabilitation of patients with oral malformationions psychological built up. We shoud recognize the patient at early stage and should provide close follow up appropriate therapy and further councelling regarding next generation.

Many people think that persons with TCS 'may not be normal as they don't look normal'. Most affected people are self conscious about their physical appearance and often put too much importance on other's perceptions. Thus there is a need to build a collaborative relationship between docters and families of affected individuals so as to promote an understanding and accepting environment for the child to live in.

\section{REFERENCES}

[1]. Eeraveni R., Singh B D, Kamarthi N, Patel M, JainM, Oral and Systemic Manifestations of Treacher Collins- Franceschetti Syndrome (Mandibulofacial Dysostosis): A Case Report. JIDA,Dec4(12),2010,545-546.

[2]. Neville BW, Damm DD,Allen CM, Bouquot JE, Developmental defects of the oral and maxillofacial region, in Oral and maxillofacial pathology(second edition).42-43.

[3]. Martelli H, Ricardo D, Roseli T. M, Orofacial features of Treacher Collins, Med Oral Cir Bucal, July1,14(7)2009,344348

[4]. Lungarotti, M. S., Marinelli, D., Mariani, T., Calabro, A. Multiple congenital anomalies associated with apparently normal maternal intake of vitamin A: a phenocopy of the isotretinoin syndrome? Am. J. Med. Genet, 27,1987, 245-248.

[5]. Marsh, K. L., Dixon, J., Dixon, M. J, Mutations in the Treacher Collins syndrome gene lead to mislocalization of the nucleolar protein treacle. Hum. Molec. Genet., 7,1998,1795-1800.

[6]. Li, C., Mernagh, J., Bourgeois, J, Novel craniofacial and extracraniofacial findings in a case of Treacher Collins syndrome with a pathogenic mutation and a missense variant in the TCOF1 gene. Clin. Dysmorph, 18,2009, 63-66.

[7]. Bowman, M., Oldridge, M., Archer, C., O'Rourke, A., McParland, J., Brekelmans, R., Seller, A., Lester, T, Gross deletions in TCOF1 are a cause of Treacher-Collins-Franceschetti syndrome. Europ. J. Hum. Genet, 20,2012, 769-777.

[8]. Shafer WG,Hine HK, Barnet M. Levy. Diseases of bone and joints, in A Textbook of Oral Pathology , (fourth edition, Saunders Elsevier Publication,2009) 681-682.

[9]. Murdoch-kinch CA. Developmental disturbances of the face and jaws, in White SC, Pharoah MJ. Oral Radiology principles and interpretation ( fifth edition) 643-646.

[10]. Shete p, Tupkari JV, Benjamin T,Singh A, Treacher Collins syndrome.JOMP, Sep-Dec,15(3),2011, 348-351.

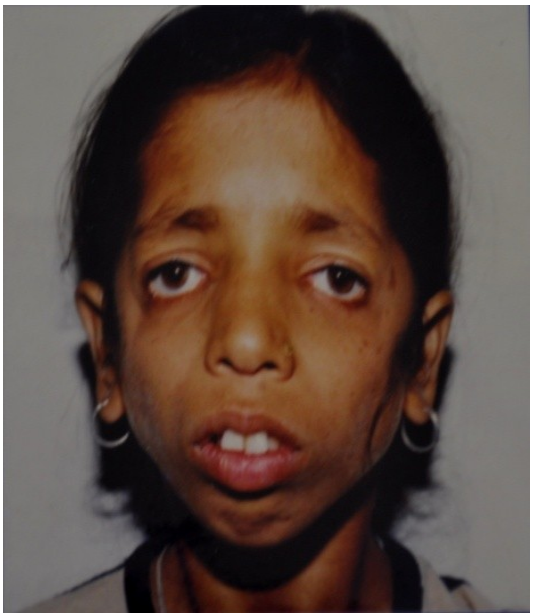

Fig. 1 characteristic bird face,downsloping palpebral fissures 


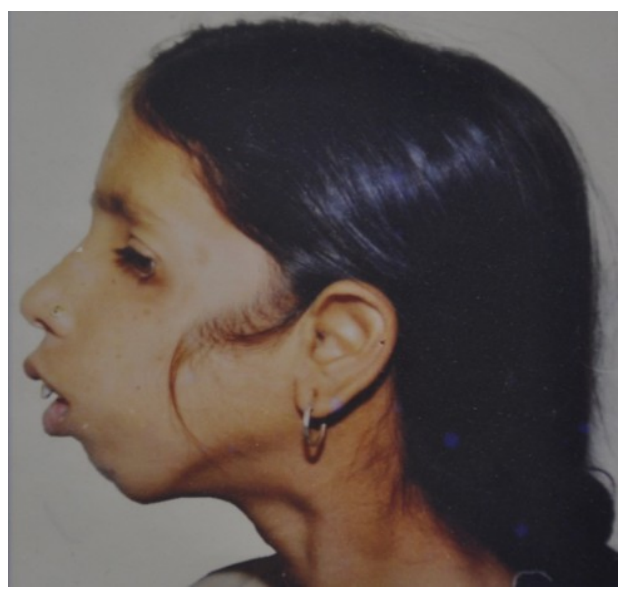

Fig. 2 showing hairline extending towards cheek, covex profile, incompetent lips and concave undersurface of body of mandible.

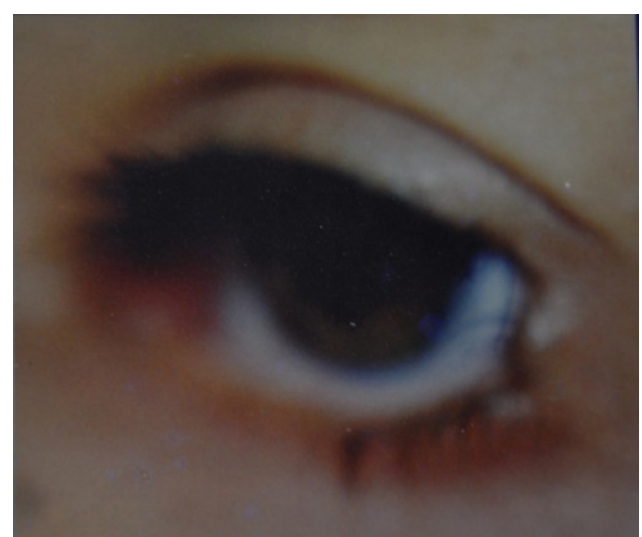

Fig. 3 coloboma in the outer third of lower lid with deficient cillia medial to it.

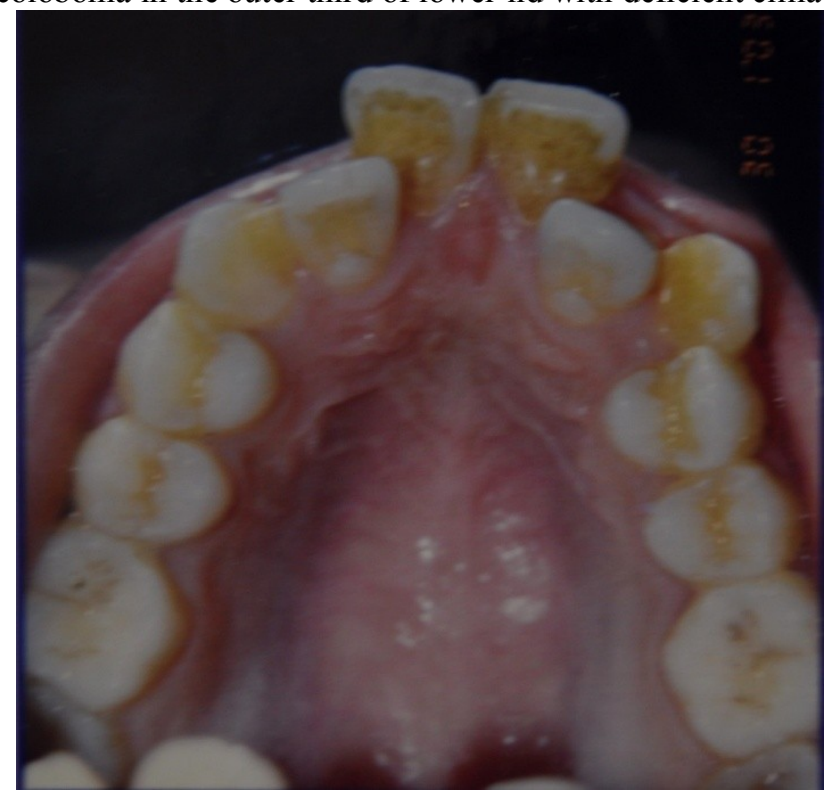

Fig4a 


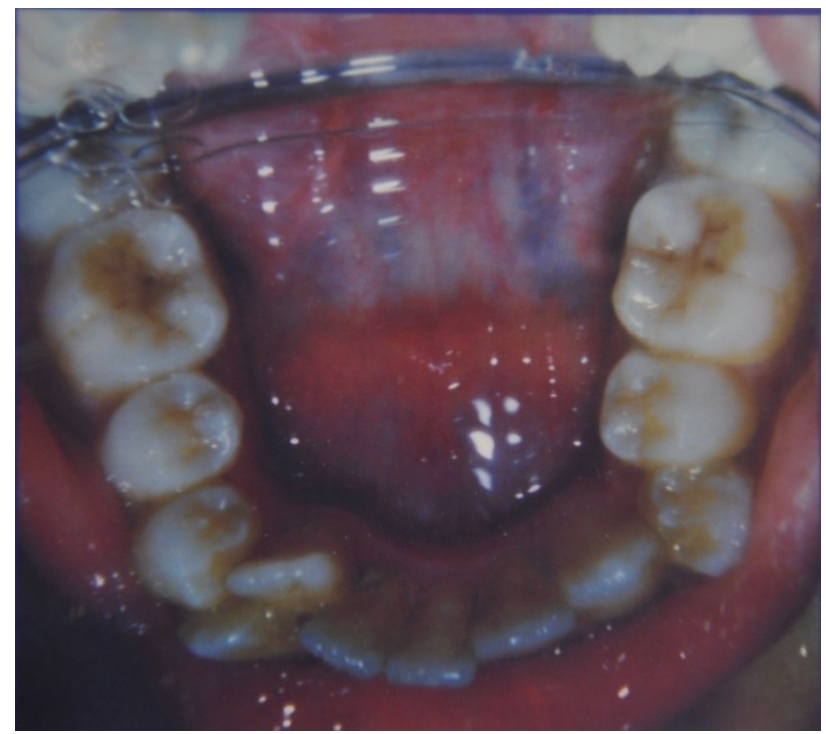

Fig. $4 \mathrm{~b}$

Fig. 4a,b- shows high arched palate, crowding in upper and lower dental arches.

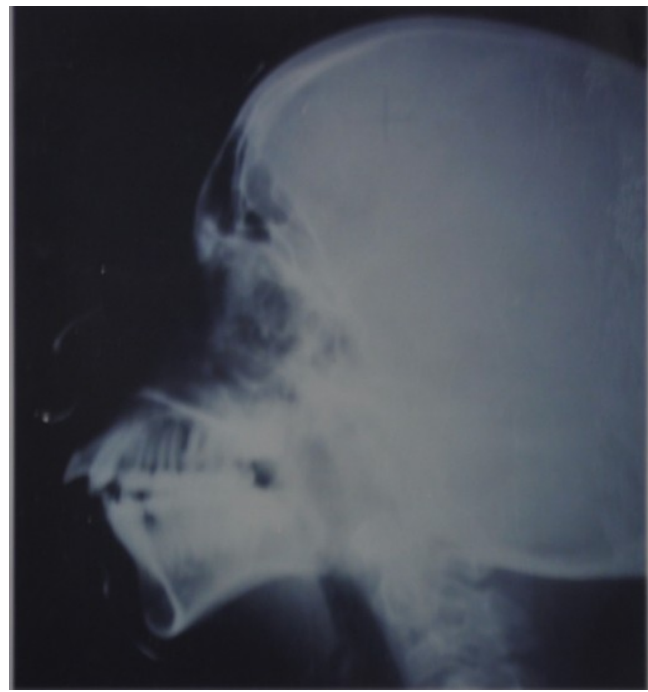

Fig. 5 hypoplastic mandible, prominent antegonial notch, acute nasolabial angle

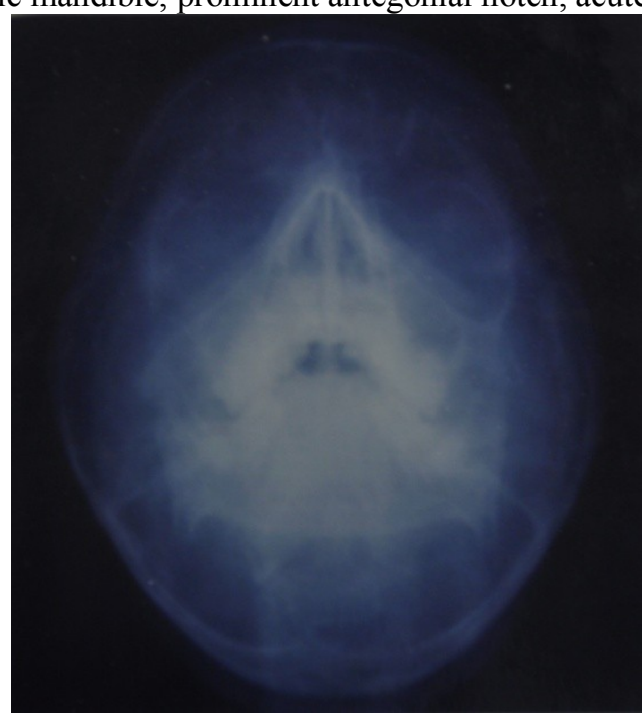

Fig. 6 underdeveloped maxillary sinuses, malar bones and hyperteloric orbits. 


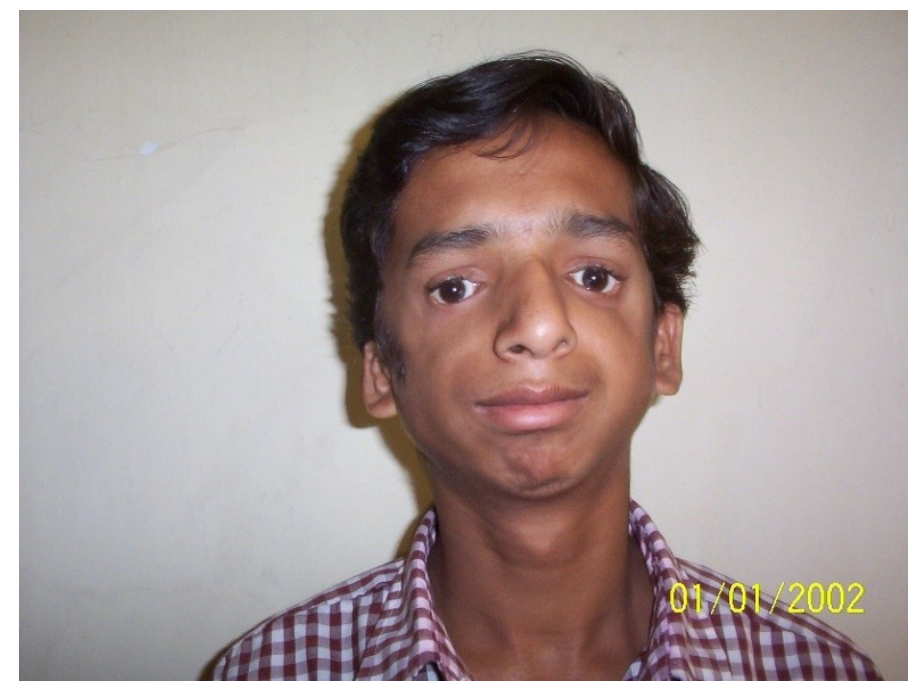

Fig.7 showing bird face with sunken malar bones, hypertelorism.

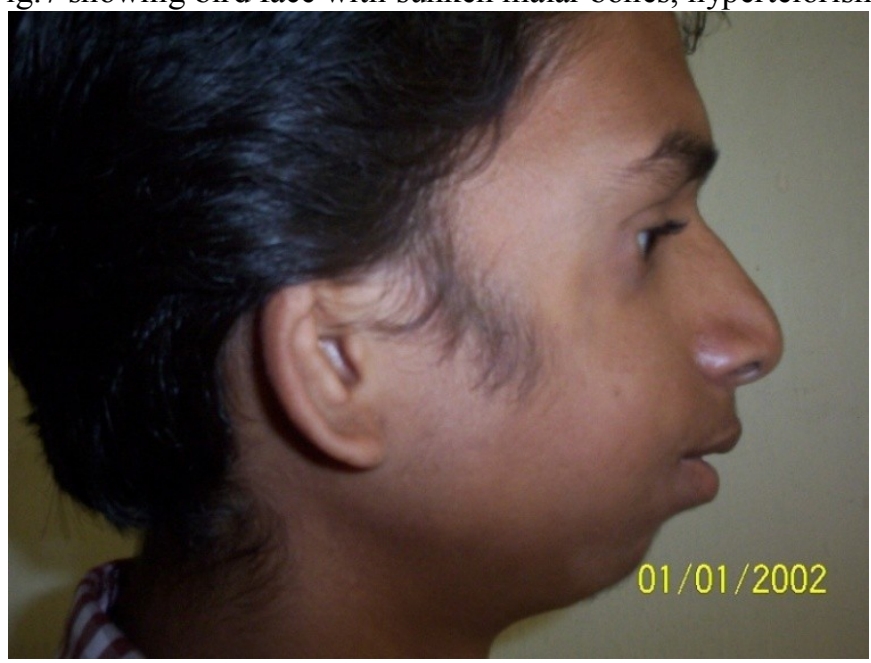

Fig 8 external ear crumpled forwardly, tongue like hair growth 